\title{
Influence of hard alloys CEDG modes on the size of dissolved layer
}

\author{
Olga Medvedeva ${ }^{1}$, Pavel Arkhipov ${ }^{1, *}$, and Alexander Yanyushkin ${ }^{2}$ \\ ${ }^{1}$ Bratsk State University, 40, Makarenko, Bratsk, 665709, Russian Federation \\ ${ }^{2}$ Chuvash State University, 15, Moskovskii, Cheboksary, 428015, Russian Federation
}

\begin{abstract}
Correctly assigned technological modes of processing define the accuracy of forming, optimum mechanical and electrochemical speed of the processed material removal, quality and durability of the cutting tool as well as provide the self-sharpening conditions for the grinding wheel. The purpose of this work is to increase the efficiency of hard alloys combined electric diamond grinding (CEDG) by establishing a dependence between the amount of material removed by mechanical cutting and the depth of the layer dissolved by electrolytic processes. Achieving the stated goal allows to solve the problem of defects occurrence on the processed surface and to minimize economic expenses for machine-building production processing. The article presents the results of mathematical calculations of the parameters influence on the size of the dissolved surface layer, as well as the current dependent removal rate for hard alloys. We determine qualitative and quantitative characteristics of the hard alloy dissolved layer dependence on the mechanical and electrical parameters of CEDG, such as the depth of grinding and anode current density, and demonstrate their analytical dependence.
\end{abstract}

\section{Introduction}

Metal bond grinding wheels demonstrate optimal results at processing the workpieces made of hard alloys and high speed steels $[1-4]$. A significant obstacle to these wheels wide application is the rapid clogging of the cutting surface. As a consequence, this affects the quality of processing. Owing to the increase in the use of hard alloys and other tough materials in the industry, there is a demand for their new efficient processing technologies. This purpose facilitates special grinding methods application [5-8].

Electrochemical methods of grinding are based on the principle of local anode dissolution in a flowing electrolyte, with anode being the processed tool or a workpiece. The processes of electro-chemical and mechanical machining of metals and alloys are based on the principle of combined removal of the surface layer by anode dissolution and mechanical destruction of the metal surface $[9,10,11]$. These processes are accompanied by various structural defects formed on the processed material surface because of the abrasive grains action at elastic-plastic deformations. However, grinding is carried out in

\footnotetext{
* Corresponding author: pavded@yandex.ru
} 
the conditions of reduced resistance to plastic deformation at selective dissolution of different hard alloys phases.

The reactions of oxides, oxygen and other gases formation can occur on the anode in addition to the reactions of the anode dissolution of metal under the electric current influence, while the isolation of hydrogen and the deposition of cations from the electrolyte solution occur on the cathode. All these factors reduce the proportion of anode dissolution. Therefore, all correlations using calculations of electrochemical parameters should be adjusted by the current-dependent removal rate coefficient $[11,12,13]$.

\section{Materials and research methods}

The research is carried out for the operations of surface grinding by the wheel periphery on universal 3E642E grinding-and-cutting machine modernized for CEDG. Metal bond diamond cup-shaped wheels were used in the experiments: ASV 80/63 M1 100\%, ASV 125/100 M1 100\%. Samples were made of the VK3, T15K6, TM3, TN20 hard alloys. Table 1 gives the values of electrochemical equivalents and densities for the studied alloys.

Table 1. Alloys properties

\begin{tabular}{|c|c|c|c|c|c|}
\hline Alloy & & VK3 & T15K6 & TM3 & TN20 \\
\hline$\varepsilon_{\mathrm{m}}, \mathrm{g} / \mathrm{A} \cdot \mathrm{min}$ & & 0.015 & 0.012 & 0.0075 & 0.0073 \\
\hline$\rho, \mathrm{g} / \mathrm{cm}^{3}$ & & 15.0 & 11.0 & 5.9 & 5.7 \\
\hline
\end{tabular}

The current dependent removal rate of material $\eta$ was defined as the deviation degree of the substance mass which actually reacted on the electrode $\left(m_{\text {pract }}\right)$ in correlation with the one theoretically calculated by Faraday's Law ( $m_{\text {theor }}$ )

$$
\eta=\frac{m_{\text {pract }}}{m_{\text {theor }}} .
$$

According to Faraday's Law the mass of the metal deposited as a result of electrochemical dissolution from a surface unit is determined by:

$$
m_{\text {theor }}=\varepsilon_{m} \cdot I \cdot T
$$

where $\varepsilon_{m}$ - is the weight electrochemical equivalent, $[\mathrm{g} / \mathrm{A} \cdot \mathrm{min}] ; I-$ is current power, [A ]; $T$ - is the time of the anode dissolution, [ $\min$ ].

Dividing this expression by the time of dissolution, multiplying and dividing by the value of the anode area, the result is:

$$
\frac{m_{\text {theor }}}{T}=\frac{\varepsilon_{m} \cdot I \cdot T}{T \cdot S_{A}} \cdot S_{A}
$$

The current dependent removal rate is also defined as the relationship of the experimentally found mass removal (substance removal rate) $Q_{m \text { exp }}$ to $Q_{m}$ theor specified theoretically [13]. 


$$
\eta=\frac{Q_{m \exp }}{Q_{m \text { theor }}} .
$$

Taking into account $(2,3)$ the expression for the definition of mass removal is the following:

$$
Q_{m \text { theor }}=\varepsilon_{m} \cdot i_{e c d} \cdot S_{A}
$$

where $i_{e c d}-$ is etching current density, $\left[\mathrm{A} / \mathrm{cm}^{2}\right] ; S_{A}-$ is the anode area, $\left[\mathrm{cm}^{2}\right]$.

The values of mass material removal from the surface unit were calculated theoretically $Q_{m \text { theor }}[\mathrm{g} / \mathrm{min}]$ for hard alloys by the formula (5). For the same alloys, the values of mass removal $Q_{m} \exp [\mathrm{g} / \mathrm{min}$ ] were obtained by experimental means [13].

\section{Results and discussion}

According to the data obtained by the formula (4) the current dependent removal rate values are determined for hard alloys at different values of anode current density $i_{e c d}$. Table 2 shows the results of the calculations.

Table 2. Current dependent removal rate values for hard alloys

\begin{tabular}{|c|c|c|c|c|}
\hline Alloy & $i_{\text {ecd }},\left[\mathrm{A} / \mathrm{cm}^{2}\right]$ & $Q_{m \text { theor }},[\mathrm{g} / \mathrm{min}]$ & $Q_{m \text { exp }},[\mathrm{g} / \mathrm{min}]$ & $\eta$ \\
\hline \multirow{4}{*}{ VK3 } & 10 & 0.5 & 0.3 & 0.60 \\
\cline { 2 - 5 } & 20 & 1.0 & 0.6 & 0.62 \\
\cline { 2 - 5 } & 30 & 1.5 & 0.9 & 0.60 \\
\cline { 2 - 5 } & 40 & 2.0 & 0.1 & 0.50 \\
\hline \multirow{4}{*}{ T15K6 } & 60 & 3.0 & 1.4 & 0.47 \\
\cline { 2 - 5 } & 10 & 1.4 & 0.9 & 0.56 \\
\cline { 2 - 5 } & 20 & 2.8 & 1.6 & 0.57 \\
\cline { 2 - 5 } & 30 & 4.1 & 2.2 & 0.53 \\
\hline \multirow{4}{*}{ TM3 } & 40 & 5.6 & 2.5 & 0.44 \\
\cline { 2 - 5 } & 60 & 8.4 & 2.7 & 0.32 \\
\cline { 2 - 5 } & 10 & 0.7 & 0.4 & 0.57 \\
\cline { 2 - 5 } & 20 & 1.4 & 1.2 & 0.85 \\
\hline \multirow{7}{*}{ TN20 } & 30 & 2.1 & 2.0 & 0.95 \\
\cline { 2 - 5 } & 40 & 2.8 & 2.3 & 0.82 \\
\cline { 2 - 5 } & 60 & 4.2 & 3.0 & 0.71 \\
\cline { 2 - 5 } & 10 & 0.7 & 0.5 & 0.74 \\
\cline { 2 - 5 } & 20 & 1.4 & 1.1 & 0.78 \\
\hline & 30 & 2.1 & 1.4 & 0.67 \\
\hline
\end{tabular}

The unstable dynamics of the change in the anode current density is explained by the fact that the high rate of material dissolution in the electrochemical cell leads to the change in the electrolyte concentration. The solution is saturated with ions of hard alloy. The dissolution of the metal results in saturation of the treated surface with carbides which reduces the material's capacity to electrochemical dissolution [11].

The current density is to be raised to a certain value in order to increase the anode removal of metal. Excessive bond dissolution leads to the softening of the hard alloy deep layers which negatively affects the quality of the workpiece processed by grinding. The 
depth of dissolution is the parameter characterizing the quantitative properties of electrochemical treatment meeting the requirements for the quality of the treated surface.

The amount of the anode-dissolved material is estimated by the amount of the dissolved layer removed from the surface unit [11]:

$$
h=\varepsilon_{h} \cdot \eta \cdot \int_{0}^{\tau} i_{e c d} d \tau
$$

where $h-$ is the size of the dissolved material layer, [ $\mathrm{mm}] ; \eta-$ is current dependent material removal rate; $\varepsilon_{h}-$ is linear electrochemical equivalent, [ $\left.\mathrm{mm} / \mathrm{A} \cdot \mathrm{min}\right] ; \tau-$ is time, [ $\min ]$.

Given that the density of the anode current is actually etching current, it does not depend on the time of dissolution owing to its small value $\left(T=10^{-5} \ldots 10^{-6} \mathrm{sec}\right)[11]$ and $T=\int_{0}^{\tau} d \tau$, the size of the dissolved layer removed from the surface of the workpiece of $S_{A}$ area is determined by the formula:

$$
h=\varepsilon_{h} \cdot \eta \cdot T \cdot S_{A} \cdot i_{e c d}
$$

where $T-$ is the time spent on dissolving, [ $\mathrm{min}$ ].

The correlation between linear and weight electrochemical equivalents is used to define $h:$

$$
\varepsilon_{h}=\frac{\varepsilon_{m} \cdot 10}{\rho \cdot S_{A}}
$$

where $\rho-$ is the material density, $\left[\mathrm{g} / \mathrm{cm}^{3}\right]$.

Taking into account (8) the formula (7) is:

$$
h=\frac{\eta \cdot \varepsilon_{m} \cdot i_{e c d} \cdot T \cdot 10}{\rho}
$$

The time spent on dissolving the surface of the processed material considering the depth of grinding, wheel radius and wheel rotation speed is determined by the formula (10) [13]:

$$
T=\frac{l}{60 \cdot V_{w h}}=\frac{R}{60 \cdot V_{w h}} \cdot \arccos \frac{R-t}{R},
$$

where $l-$ is the length of the contact surface, $[\mathrm{mm}]$;

$t-$ is the depth of grinding, [ $\mathrm{mm}]$;

$R$ - is the radius of the grinding wheel, [ $\mathrm{mm}]$;

$V_{w h}$ - is the rotation speed of the grinding wheel, [ $\left.\mathrm{m} / \mathrm{s}\right]$.

In fact, the depth of substance dissolution is determined considering $(7-10)$ by the formula:

$$
h=\frac{\eta \cdot \varepsilon_{m} \cdot i_{e c d} \cdot R \cdot \arccos \frac{R-t}{R}}{\rho \cdot V_{w h} \cdot 6000}
$$


The dependence of the dissolution depth of alloys on the grinding depth is shown in the diagrams of Fig. $1-4$.

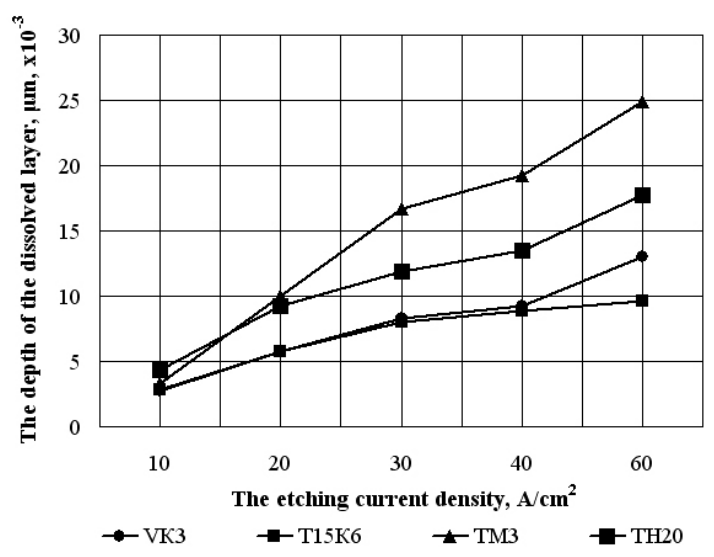

Fig. 1. The dissolution layer depth of various alloys depending on the etching current density of $V_{w h}=$ $35 \mathrm{~m} / \mathrm{s}, i_{e c d}=10 \ldots 60 \mathrm{~A} / \mathrm{cm}^{2}, t=0.01 \mathrm{~mm}$.

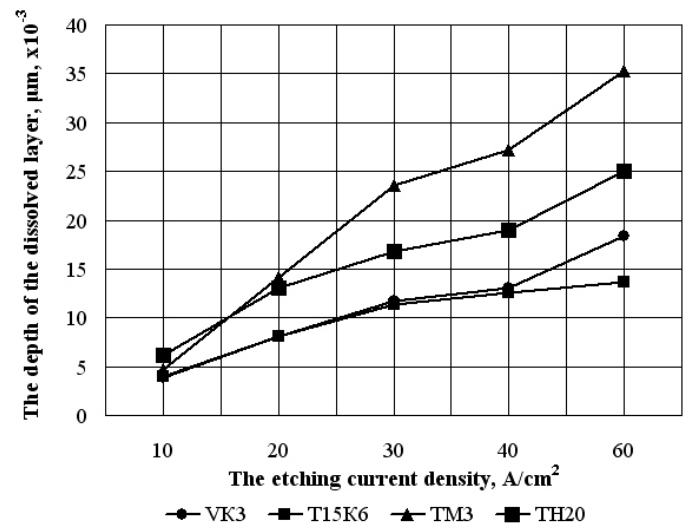

Fig. 2. The dissolved layer depth of different alloys depending on the etching current density of $V_{w h}=$ $35 \mathrm{~m} / \mathrm{s}, i_{e c d}=10 \ldots 60 \mathrm{~A} / \mathrm{cm}^{2}, t=0.02 \mathrm{~mm}$.

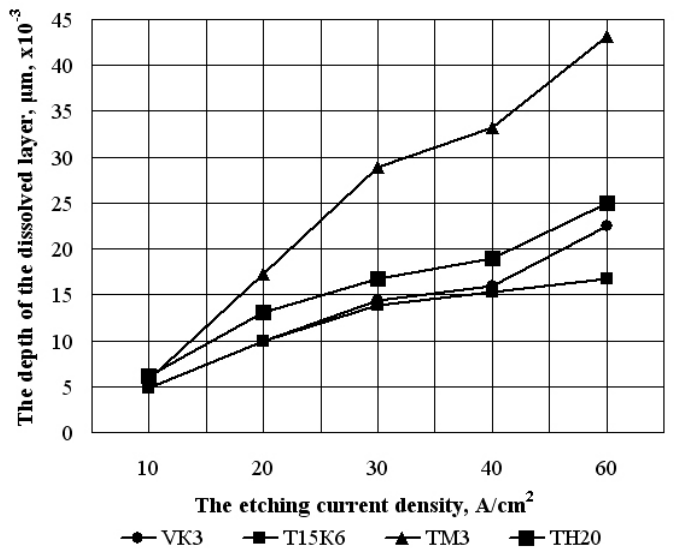

Fig. 3. The dissolved layer depth of different alloys depending on the etching current density of $V_{w h}=$ $35 \mathrm{~m} / \mathrm{s}, i_{e c d}=10 \ldots 60 \mathrm{~A} / \mathrm{cm}^{2}, t=0.03 \mathrm{~mm}$. 


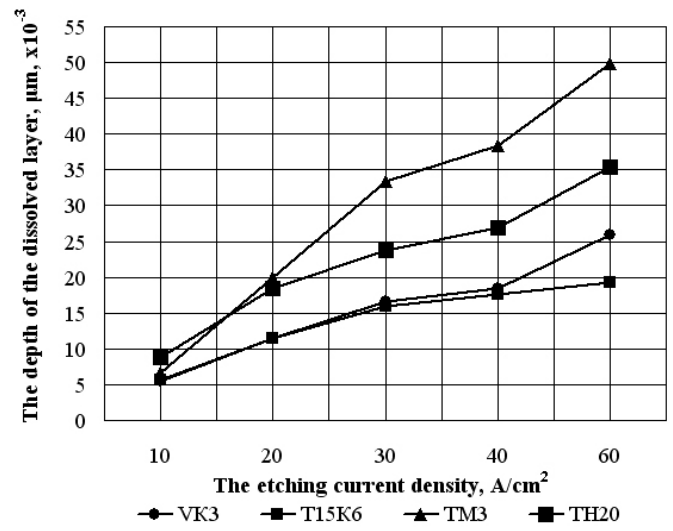

Fig. 4. The dissolved layer depth of different alloys depending on the etching current density of $V_{w h}=$ $35 \mathrm{~m} / \mathrm{s}, i_{\text {ecd }}=10 \ldots 60 \mathrm{~A} / \mathrm{cm}^{2}, t=0.04 \mathrm{~mm}$.

The depth of dissolution essentially depends on the depth of grinding t. According to the formula (9), with the increase of $t$ the time of electrochemical impact on the surface unit increases. It is noteworthy that $h=h(t)$ is not a linear dependence. According to the results shown in table 3 , the doubling of the grinding depth $t$ (increase from 0.01 to 0.02 ) leads to an $1.2-1.4$ times increase in the depth of dissolution $h$, and a fourfold increase in $t$ (from 0.01 to 0.04 ) leads to an $1.7-2$ increase in $h$.

The efficiency of the electro-diamond grinding depends on the contact area which is the function of the grinding depth: the greater its value, the greater the amount of electric current is required for the electrochemical dissolution. This naturally affects the processing efficiency and the amount of material removed from the treated surface.

\section{Conclusions}

The amount of material removed from the surface as a result of combined electro-diamond grinding consists of the layer mechanically ground with diamond grains and the layer dissolved in the electrolyte. The amount of the dissolved layer is used to determine the contribution of electrochemical removal to the total volume of the ground material. Correctly assigned technological mode parameters define the accuracy of forming, optimum mechanical and electrochemical speed of material removal and provide the conditions for the grinding wheel self-sharpening. The use of combined electro-diamond grinding allows optimum use of electrical phenomena for anode dissolution of the processed material surface by selecting the mechanical parameters. The research established a close connection between the depth of the dissolved layer and the depth of grinding. The choice of optimal modes of combined electro-diamond grinding solves the problem of defects formation on the treated surface, as well as minimizes the cost of workpiece machining.

The reported study was funded by RFBR according to research project №16-38-00123 mol_a.

\section{References}

1. P. Herzenstiel, J.C. Aurich, $C B N$-grinding wheel with a defined grain pattern - extensive numerical and experimental studies, Machining Science and Technology, 14, pp. 301-322 (2010). 
2. Zhi Geng, Li Xuekun, Qian Zhiqiang, Liu Haitao, Rong Yiming, Experimental study of time-dependent performance in superalloy high-speed grinding with CBN wheels, Machining Science and Technology, Vol. 20(4), pp. 615-633 (2016).

3. E.V. Vasil'ev, A.Y. Popov, D.S. Rechenko, Diamond grinding of hard-alloy plates, Russian Engineering Research, 32(11-12), pp. 730-732 (2012).

4. Z.I. Kremen', D.A. Popovskii, V.G. Yur'ev, Grinding of titanium alloys by Elbor and diamond wheels, Russian Engineering Research, 33, pp. 457-459 (2013).

5. V.A. Mogilnikov, M.Y. Chmir, Y.S. Timofeev, V.S. Poluyanov, Diamond-ECM Grinding of Sintered Hard Alloys of WC-Ni, Procedia CIRP, 42, pp. 143-148 (2016).

6. Andre D.L. Batako, Vaios Tsiakoumis, An experimental investigation into resonance dry grinding of hardened steel and nickel alloys with element of $M Q L$, The International Journal of Advanced Manufacturing Technology, 77, pp. 27-41 (2015).

7. D. Zishan, L. Beizhi, L.Y. Steven, Material phase transformation at high heating rate during grinding, Machining Science and Technology, 20, pp. 290-311 (2016).

8. V.A. Nosenko, The influence of contact processes on the wheel wear during grinding, Tools and Technology, v. 17-18, pp. 162-167 (2004).

9. A.S. Yanyushkin, D.V. Lobanov, P.V. Arkhipov, Research of influence of electric conditions of the combined electro-diamond machining on quality of grinding of hard alloys, IOP Conf. Series: Materials Science and Engineering, 91, pp. 1-6 (2015).

10. D.V. Lobanov, P.V. Arkhipov, A.S. Yanyushkin, V.Yu. Skeeba, The research into the effect of conditions of combined electric powered diamond processing on cutting power, Key Engineering Materials, Vol. 736, pp. 81-85 (2017).

11. M.V. Shcherbak, M.A. Tolstoy, A.P. Anisimov, V.H. Postanogov, Theoretical and practical fundamentals of metals and alloys electrochemical processing (Mechanical Engineering, 1981)

12. V.S. Shorkin, L.Yu. Frolenkova, A.S. Azarov, Assessing the influence of environment particles triple interaction on surface and adhesive properties of solid bodies, Materials Science, v. 2, pp. 2-7 (2011).

13. O.I. Medvedeva, A.S. Yanyushkin, V.Y. Popov, Calculation of the energy of adhesion contact surfaces for instrumental materials by various methods, High Tech in machine building, v. 15(35), pp. 14-19 (2014). 